Supporting Information for

\title{
Blue Electroluminescence from Oxadiazole Grafted Poly(Phenylene-Ethynylene)s
}

\author{
Craig A. Breen, ${ }^{\dagger, \S}$ Sandra Rifai, ${ }^{\dagger}$ Vladimir Bulovic, ${ }^{\ddagger, \S}$ and Timothy M. Swager ${ }^{*},, \S$ \\ 'Department of Chemistry, Laboratory of Organic Optics and Electronics, \\ Department of Electrical Engineering and Computer Science, and ${ }^{\S}$ Institute for \\ Soldier Nanotechnologies, Massachusetts Institute of Technology, 77 \\ Massachusetts Avenue, Cambridge MA 02139
}

\section{Experimental Section}

\section{General:}

The general synthesis of the ortho- ${ }^{1}$ and $\mathrm{para}^{2}$ monomers as well as identical procedures used for the preparation of the ungrafted polymers and ATRP macroinitiators ${ }^{9}$ described here has been previously reported. The vinyl oxadiazole was synthesized following a literature method. ${ }^{3}$ A general preparation for oxadiazole grafted PPEs as well as the characterization of the polymers used for this study can be found below. ${ }^{1} H N M R$ spectra were recorded on a Varian MERCURY (300 MHz) using deuterochloroform as a reference or an internal deuterium lock. The chemical shift data for each signal are given in units of $d(\mathrm{ppm})$ relative to tetramethylsilane (TMS) where $d(\mathrm{TMS})=0$, and are referenced to the solvent residual. Infrared spectra were collected with a Perkin Elmer 1000 FT-IR spectrometer in $\mathrm{KBr}$ and were recorded in reciprocal centimeters $\left(\mathrm{cm}^{-1}\right.$, wavenumbers). UV-visible absorption spectra were measured with a Cary $50 \mathrm{UV} /$ Visible spectrometer. Photoluminescence spectra were measured with a SPEX Fluorolog-t2 fluorometer (model FL112, 450W xenon lamp). Solution state quantum yields were determined relative to Coumarin 314 in ethanol $(\Phi=0.63)$ and quinine sulfate in $1 \mathrm{~N}$ sulfuric acid $(\Phi=0.55)$. Solid state quantum yields were determined relative to $\sim 10^{-2} \mathrm{M}$ 9,10 diphenylanthracene in PMMA $(\Phi=0.83)$. The molecular weights of polymers were determined by Gel Permeation Chromatography (GPC) running with THF as the eluent versus polystyrene standards (PolySciences) using Hewlett Packard series 1100 HPLC instrument equipped with a Plgel $5 \mathrm{~mm}$ Mixed-C (300 x $7.5 \mathrm{~mm})$ column.

\section{Polymer Synthesis:}

General ATRP polymerization of vinyl oxadiazole to give oxadiazole grafted PPEs (G1-G5): A $25 \mathrm{~mL}$ Schlenk tube was loaded with the respective PPE ATRP macroinitiator ${ }^{9}$ (40mg, $0.03 \mathrm{mmol}$ ), $\mathrm{CuBr}$ (9.5 $\mathrm{mg}, 0.07 \mathrm{mmol}$ ), and 2,2'-dipyridyl (Bipy) (26mg, $0.17 \mathrm{mmol}$ ). Approximately $750 \mathrm{mg}$ of vinyl oxadiazole ${ }^{12}$ was dissolved in $2-3$ $\mathrm{mL}$ of anhydrous toluene and then syringed into the reaction tube. The mixture was frozen, evacuated, and thawed three times. The reaction flask was then heated in an oil bath to $140{ }^{\circ} \mathrm{C}$ and allowed to stir for $72 \mathrm{~h}$. At the end of the reaction time, the reaction flask was cooled in liquid $\mathrm{N}_{2}$ in order to quench the polymerization and then the mixture 
was filtered and precipitated out of methanol. The precipitate was then filtered, washed several times with water, collected, and dried. The isolated polymer was re-dissolved in THF and precipitated out of acetone to remove any oxadiazole homopolymer produced in the reaction. The precipitate was isolated by centrifugation due to partial solubility in acetone. The remaining pellet was re-dissolved in THF and precipitated one last time out of methanol. The precipitate was filtered, collected and dried to give oxadiazole grafted PPEs.

\section{Polymer Characterization:}

1: Reported previously; ${ }^{9}$ GPC $M_{n} 2.30 \times 10^{4}, M_{w} 6.70 \times 10^{4}, M_{w} / M_{n} 2.90$.

1 ATRP macroinitiator: Reported previously. ${ }^{9}$

G1: $n_{\max } 3026,2924,2853,1725,1613,1488,1269,1067,1005,822,713$, and 558; $d_{\mathrm{H}}$ 8.2-7.8 (Aromatic br signal), 7.8-7.2 (Aromatic br signal), 7.2-6.6 (Aromatic br signal), 6.5 (br signal), 4.0 (br signal), 2.6-1.7 (br signal), 1.4 (br s), 1.2 (br s), 0.85 (br signal); GPC $M_{n} 4.10 \times 10^{4}, M_{w} 7.00 \times 10^{4}, M_{w} / M_{n} 1.70$.

2: $n_{\max } 3406,2922,2851,1636,1508,1467,1435,1377,1262,1220,1041,809$, and $721 ; d_{\mathrm{H}} 7.2(2 \mathrm{H} \mathrm{br} \mathrm{s}), 7.0(2 \mathrm{H}$ br s), $4.2(4 \mathrm{H}$, br t), $4.0(4 \mathrm{H}$, br t), $3.6(4 \mathrm{H}$, br t, $J 6.6), 1.8$ $\left(8 \mathrm{H}\right.$, br t), $1.5\left(32 \mathrm{H}\right.$, br signal), $1.3\left(52 \mathrm{H}\right.$, br signal), $0.88(6 \mathrm{H}$, br t, $J 6.6)$; GPC $M_{n} 2.80 \mathrm{x}$ $10^{4}, M_{w} 9.80 \times 10^{4}, M_{w} / M_{n} 3.5$.

2 ATRP macroinitiator: $n_{\max }$ 2924, 2852, 1736, 1508, 1466, 1434, 1371, 1275, 1220, $1162,1109,1029,859,816$, and $721 ; d_{\mathrm{H}} 7.0$ (br s), 4.2 (br signal), 4.0 (br t), 1.9 (s), 1.8 (br signal) 1.6-1.1 (br signal), 0.9 (br t); GPC $M_{n} 5.20 \times 10^{4}, M_{w} 1.40 \times 10^{5}, M_{w} / M_{n} 2.70$.

G2: $n_{\max } 3027,2923,2851,1725,1612,1487,1267,1067,1005,822,712$, and 557; $d_{\mathrm{H}}$ 8.2-7.8 (Aromatic br signal), 7.8-7.2 (Aromatic br signal), 7.2-6.6 (Aromatic br signal), 6.5 (br signal), 4.1 (br multiplet), 2.2-1.9 (br signal), 1.8 (br signal), 1.4 (br s), 1.2 (br s), 0.86 (br signal); GPC $M_{n} 4.70 \times 10^{4}, M_{w} 7.50 \times 10^{4}, M_{w} / M_{n} 1.60$.

3: $n_{\max } 3340,2919,2850,1506,1468,1438,1378,1225,1192,1088,807$, and $720 ; d_{\mathrm{H}}$ 7.2 (4H, br s), $4.2(8 \mathrm{H}$, br t), $3.6(4 \mathrm{H}$. br t, $J 6.6), 1.8(8 \mathrm{H}$, br q), $1.5(32 \mathrm{H}$, br signal), 1.3 (52H, br signal), $0.88(6 \mathrm{H}$, br t, $J 6.6)$; GPC $M_{n} 1.40 \times 10^{4}, M_{w} 3.22 \times 10^{4}, M_{w} / M_{n} 2.30$.

3 ATRP macroinitiator: $n_{\max } 2919,2850,1736,1577,1503,1467,1438,1378,1275$, $1225,1191,1164,1108,1087,1027,808$, and $721 ; d_{\mathrm{H}} 7.2(4 \mathrm{H}$, br s), $4.16(4 \mathrm{H}$, br t, $J$ 6.6), $4.0(8 \mathrm{H}$, br t), $1.9(12 \mathrm{H}$, br s), $1.8(8 \mathrm{H}$, br q), $1.7(8 \mathrm{H}$, br q), $1.5(12 \mathrm{H}$, br signal), 1.4-1.2 (64H, br signal), 0.9 (6H, t, $J$ 6.6).

G3: $n_{\max } 3026,2923,2851,1724,1613,1487,1268,1067,1005,821,712$, and 557; $d_{\mathrm{H}}$ 8.2-7.8 (Aromatic br signal), 7.8-7.2 (Aromatic br signal), 7.2-6.6 (Aromatic br signal), 6.5 (br signal), 4.1 (br signal), 2.0 (br signal), 1.8 (br signal), 1.4 (br s), 1.2 (br s), 0.85 (br s); GPC $M_{n} 3.10 \times 10^{4}, M_{w} 4.70 \times 10^{4}, M_{w} / M_{n} 1.50$.

4: $n_{\max } 3422,3068,3021,2924,2852,1500,1458,1381,1279,1198,1023,754,673$, and $568 ; d_{\mathrm{H}} 7.5(6 \mathrm{H}$, br signal), $7.05(4 \mathrm{H}$, br signal), $6.1(4 \mathrm{H}$, br s), $4.5(4 \mathrm{H}$, br t), 3.6 (4H, br t, $J$ 6.6), 2.2 (4H, br signal), 1.7 (4H, br signal), 1.47 (8H, br signal), $1.2(28 \mathrm{H}$, br signal); GPC $M_{n} 2.90 \times 10^{4}, M_{w} 9.30 \times 10^{4}, M_{w} / M_{n} 3.20$.

4 ATRP macroinitiator: $n_{\max } 3069,3021,2925,2853,1734,1502,1458,1414,1383$, $1277,1213,1163,1107,1023,860,803,754,673$, and $569 ; d_{\mathrm{H}} 7.5(6 \mathrm{H}$, br signal $), 7.05$ (4H, br signal), $6.12(4 \mathrm{H}$, br s), $4.5(4 \mathrm{H}$, br t), 4.1 (4H, br t, J 6.6), 2.2 (4H, br signal), 1.9 $(12 \mathrm{H}$, br s), $1.8(6 \mathrm{H}$, br q), $1.6(8 \mathrm{H}$, br multiplet $), 1.4(8 \mathrm{H}$, br signal $), 1.3-1.0(18 \mathrm{H}$, br signal). 
G4: $n_{\max } 3024,2959,2857,1723,1612,1487,1267,1096,1005,821,712$, and 557; $d_{\mathrm{H}}$ 8.2-7.8 (Aromatic br signal), 7.8-7.2 (Aromatic br signal), 7.2-6.6 (Aromatic br signal), 6.5 (br signal), 6.1 (br signal), 4.4 (br signal), 4.0 (br signal), 2.4-1.5 (br signal), 1.3 (br s), 1.1 (br s); GPC $M_{n} 4.50 \times 10^{4}, M_{w} 7.70 \times 10^{4}, M_{w} / M_{n} 1.70$.

5: $n_{\max } 3407,3068,3021,2925,2852,1492,1458,1429,1380,1319,1228,1198,1179$, 1056, 754, 673, and 567; $d_{\mathrm{H}} 7.5(6 \mathrm{H}$, br signal), $7.05(4 \mathrm{H}$, br signal), $6.07(4 \mathrm{H}$, br s), 4.6 (4H, br signal), 3.6 (4H, br t, $J 6.6), 2.17(4 \mathrm{H}$, br signal), $1.6(4 \mathrm{H}$, br signal), $1.5(8 \mathrm{H}$, br multiplet), 1.4-1.0 (28H, br signal); GPC $M_{n} 5.50 \times 10^{4}, M_{w} 1.40 \times 10^{5}, M_{w} / M_{n} 2.50$.

5 ATRP macroinitiator: $n_{\max } 3068,3020,2926,2852,2679,1732,1494,1458,1430$, $1370,1275,1228,1163,1108,1023,814,754,673$, and $568 ; d_{\mathrm{H}} 7.5(6 \mathrm{H}$, br signal), 7.1 (4H, br signal), 6.05 (4H, br s), 4.6 (4H, br t), 4.15 (4H, br t, $J$ 6.6), 3.1 (4H, br signal), $2.1(4 \mathrm{H}$, br signal), $1.9(12 \mathrm{H}$, br s), $1.6(6 \mathrm{H}$, br q), $1.5(6 \mathrm{H}$, br multiplet $), 1.3(8 \mathrm{H}$, br signal), 1.1 (16H, br signal).

G5: $n_{\max } 3024,2925,2853,1723,1612,1487,1265,1096,1005,821,712$, and 567; $d_{\mathrm{H}}$ 8.2-7.8 (Aromatic br signal), 7.8-7.2 (Aromatic br signal), 7.2-6.6 (Aromatic br signal), 6.5 (br signal), 6.0 (br signal), 4.6 (br signal), 4.0 (br signal), 3.5 (br signal), 2.2 (br signal), 1.6 (br signal), 1.4 (br s), 1.2 (br signal); GPC $M_{n} 9.80 \times 10^{4}, M_{w} 1.80 \times 10^{5}$, $M_{w} / M_{n} 1.80$.

\section{Fabrication of electroluminescent devices:}

The devices were built on ITO coated glass substrates with a sheet resistance of $20 \Omega$ $\mathrm{cm}^{-2}$. The ITO glass was cleaned by ultrasonic agitation in detergent solution, rinsed with DI water, acetone, boiled in trichloroethane, rinsed with acetone, boiled in isopropyl alcohol, and $\mathrm{N}_{2}$ blown dry. Immediately prior to material deposition, substrates were pretreated with UV/Ozone for 5 minutes. The devices were fabricated by first spincasting (Velocity: $2.5 \mathrm{~K}$ revolutions $\mathrm{min}^{-1}$; Acceleration: $10 \mathrm{~K}$ revolutions $\min ^{-1} \mathrm{sec}^{-1}$; Duration: $60 \mathrm{sec}$.) the PEDOT:PSS layer, followed by thermal annealing at $175{ }^{\circ} \mathrm{C}$ for 5 minutes. Next, the oxadiazole-grafted PPE layer is spun-cast (Velocity: $3 \mathrm{~K}$ revolutions $\min ^{-1}$; Acceleration: $10 \mathrm{~K}$ revolutions $\mathrm{min}^{-1} \mathrm{sec}^{-1}$; Duration: $60 \mathrm{sec}$.) followed by vacuum deposition of $\mathrm{Ag}: \mathrm{Mg}(100 \mathrm{~nm} ; 10: 1$ by weight), and $\mathrm{Ag}(30 \mathrm{~nm})$. A shadow mask with 1 $\mathrm{mm}^{2}$ openings was used to define the metal electrodes. The EL spectra were recorded on an Acton Research Spectra Pro 300i spectrometer. The luminance-voltage and currentvoltage characteristics were measured on an Agilent-4156C Precision Semiconductor Parameter Analyzer using a Newport 818-UV photodetector. All measurements conducted at room temperature under ambient atmosphere.

\section{References:}

(1) Zhu, Z. G.; Swager, T. M. Org. Lett. 2001, 3, 3471.

(2) Breen, C. A.; Deng, T.; Breiner, T.; Thomas, E. L.; Swager, T. M. J. Am. Chem. Soc. 2003, 125, 9942.

(3) Kurfurst, A.; Lhotak, P.; Nadenik, P.; Raclova-Pavlicova, F.; Kuthan, J. Collect. Czech. Chem. Commun. 1991, 56, 1495. 